\title{
Cambios en el complejo de células ganglionares de la retina y la presión intraocular durante la gestación, medidos por tomografía de coherencia óptica y tonómetro de aplanación de Goldmann
}

\section{Changes in the retinal ganglion cell complex and intraocular pressure during pregnancy measured by optical coherence tomography and Goldman applanation tonometer}

\author{
Karen Analí García Medina ${ }^{1 *}$, Ricardo Daniel Chávez Gutiérrez² ${ }^{2}$ Felipe de Jesús Peraza Garay ${ }^{3}$ \\ 1. Residente de tercer año de Oftalmología General, Hospital Civil de Culiacán/Centro de Investigación y Docencia en Ciencias \\ de la Salud. \\ 2. Cirujano Oftalmólogo con alta especialidad en Glaucoma, Hospital Civil de Culiacán/Centro de Investigación y Docencia en \\ Ciencias de la Salud \\ 3. Doctor en Ciencias con orientación en probabilidad y estadística, Centro de Investigación y Docencia en Ciencias de la Salud
}

*Autor de correspondencia: Karen Analí García-Medina, Calle Álvaro Obregón 1763, Colonia Tierra Blanca, CP. 80030

DOI http://dx.doi.org/10.28960/revmeduas.2007-8013.v12.n1.006

Recibido 02 de marzo 2021, aceptado 16 de agosto 2021

\section{RESUMEN}

Objetivo: Determinar los cambios en la presión intraocular y complejo de células ganglionares en los tres trimestres del embarazo y en pacientes controles no embarazadas. Material y Métodos: Se analizaron ambas variables (presión intraocular y complejo de células ganglionares de la retina) en un grupo de pacientes embarazadas a quienes se les realizaron mediciones cada trimestre de la gestación para posteriormente comparar los resultados con los de pacientes sanas no gestantes valoradas en tres trimestres consecutivos. De acuerdo con una prueba de equivalencia de medias se requirió una muestra de tamaño $n=37$ en el grupo de referencia y en el grupo control, con una potencia de $80 \%$ y una significancia del $5 \%$. Se utilizó la base de datos del programa SPSS para la organización, codificación y análisis estadístico de los resultados. Resultados: los hallazgos de estos estudios difieren con lo descrito en la literatura en los que se mencionan una tendencia hacia la disminución de la presión intraocular; en este trabajo no se presentó variación clínica ni estadísticamente significativa durante la gestación en comparación con las pacientes no gestantes. En el complejo de células ganglionares de la retina tampoco se observó una variación clínica ni estadísticamente significativa.

Conclusiones: Las pacientes estudiadas no contaban con diagnóstico de glaucoma, lo cual hace que los resultados no sean extrapolables a un grupo con la patología, pero permiten conocer la evolución del complejo de células ganglionares de la retina y la presión intraocular durante la gestación, cuestión importante ya que existen pocos estudios que analicen esta información.

Palabras Clave: Gestación, glaucoma, presión intraocular, complejo de células ganglionares.

\section{ABSTRACT}

Objective: To determine the changes in intraocular pressure and ganglion cell complex in the three trimesters of pregnancy and in nonpregnant control patients. Material and Methods: Both variables (intraocular pressure and retinal ganglion cell complex) were analyzed in a group of pregnant patients who underwent measurements each trimester of pregnancy to subsequently compare the results with those of healthy non-pregnant patients assessed. in three consecutive quarters. According to an equivalence of means test, a sample of size $n=37$ was required in the reference group and in the control group, with a power of $80 \%$ and a significance of $5 \%$. The database of the SPSS program was used for the organization, coding and statistical analysis of the results. Results: the findings of this study differ from what is described in the literature in which a tendency towards a decrease in intraocular pressure is mentioned; In this study, there was no clinical or statistically significant variation during pregnancy compared to non-pregnant patients. Neither clinically nor statistically significant variation was observed in the retinal ganglion cell complex. Conclusions: The patients studied did not have a diagnosis of glaucoma, which means that the results cannot be extrapolated to a group with the pathology, but they allow us to know the evolution of the retinal ganglion cell complex and intraocular pressure during pregnancy, a question important since there are few studies that analyze this information.

Key words: Pregnancy, glaucoma, intraocular pressure, ganglion cell complex

\section{Introducción}

El glaucoma es por definición una neuropatía óptica anterior crónica, progresiva, multifactorial, en donde el aumento de la presión intraocular es el factor más importante a considerar y el único factor de riesgo tratable que contribuye a su control. ${ }^{1}$ La comprensión de la dinámica del humor acuoso es esencial para la evaluación del glaucoma ya que es esta sustancia la encargada de la presión intraocular.

El humor acuoso se produce en la cámara posterior y fluye a través de la pupila a la cámara anterior, posteriormente sale del ojo pasando 
por la red trabecular y el canal de Schlemm antes de drenar en el sistema venoso mediante un sistema de canales colectores, así como a través de la vía uveoescleral en donde el humor acuoso fluye a través de los músculos ciliares y hacia el espacio supracoroideo. ${ }^{2}$

La formación del humor acuoso es un proceso biológico que está sujeto a ritmos circadianos. La supresión de la formación acuosa puede realizarse mediante distintos mecanismos por la acción de diversas clases de drogas, entre ellas los inhibidores de la anhidrasa carbónica, los antagonistas adrenérgicos y los alfa 2 agonistas. $^{3}$

Los datos agrupados de grandes estudios epidemiológicos occidentales indican que la presión intraocular (PIO) media es aproximadamente de $16 \mathrm{~mm} \mathrm{Hg}$, con una desviación estándar de $3 \mathrm{~mm} \mathrm{Hg}$. Sin embargo, la PIO tiene una distribución no gaussiana con un sesgo hacia presiones más altas, especialmente en individuos mayores de 40 años. El valor de $22 \mathrm{~mm}$ $\mathrm{Hg}$ se ha utilizado en el pasado tanto para distinguir presiones normales de anormales como para definir qué pacientes requieren terapia hipotensora ocular. Esta división se basó en las suposiciones clínicas erróneas de que el daño glaucomatoso es causado exclusivamente por presiones que son más altas de lo normal y que las presiones normales no ocasionan daño. Un ejemplo de las deficiencias creadas por estos supuestos es que la detección de la enfermedad glaucomatosa basada únicamente en la PIO $>21 \mathrm{~mm} \mathrm{Hg}$ no detecta a la mitad de las personas con glaucoma en la población examinada. ${ }^{4}$

Se llegó al acuerdo generalizado de que, para la población en general, no existe nivel de PIO claro por debajo del cual puede considerarse "normal" o segura y por encima del cual la PIO puede ser considerada "elevada" o insegura: algunos ojos sufren daño con PIO de $18 \mathrm{~mm} \mathrm{Hg}$ o menos. Sin embargo, la elevación de la PIO aún se considera un factor de riesgo importante para el desarrollo del daño glaucomatoso del nervio óptico.

Aunque otros factores de riesgo afectan la susceptibilidad de un individuo al daño por la enfermedad, la PIO es el único que puede ser modificado de manera efectiva en la actualidad. ${ }^{5}$

Es conocido que algunas condiciones específicas, como el embarazo, favorecen el correcto control del glaucoma, ya sea manteniendo constante la PIO o limitando el avance de la enfermedad, sin embargo, se desconocen las modificaciones que existen durante la gestación en cuanto a la PIO y el complejo de células ganglionares de la retina. ${ }^{6}$

El objetivo de este estudio es realizar la medición de dichos parámetros en mujeres embarazadas y su comparación en cada trimestre de la gestación con pacientes sanas no gestantes. 


\section{Materiales y métodos}

Es un estudio comparativo, descriptivo, prospectivo y longitudinal que se llevó a cabo en pacientes embarazadas a quienes se les realizó medición de la PIO con tonómetro de aplanación de Goldmann y registro del complejo de células ganglionares de la retina por tomografía de coherencia óptica (TCO) con el quipo modelo Optovue RTvue, ambos parámetros fueron valorados en cada uno de los trimestres de la gestación con la finalidad de comparar los resultados obtenidos con los de un grupo control de pacientes no gestantes a quienes se les realizaron las mismas mediciones en tres trimestres consecutivos.

Tomando en cuenta que la TCO es un estudio de imagen no invasivo e interferométrico, que utiliza ondas de luz para obtener imágenes en corte transversal de la retina, que al no utilizar flashes ni precisar anestésicos se convierte en una técnica exploratoria segura, mientras que al realizar la medición de la PIO con el tonómetro de aplanación de Goldmann sólo se precisa de anestesia tópica minimizando así los posibles efectos adversos del procedimiento incluyendo una reacción alérgica a la anestesia como el efecto adverso más probable.

El seguimiento de las pacientes embarazadas se realizó con la primera valoración durante las 13 semanas iniciales de la gestación y posteriormente en el segundo y tercer trimestres del embarazo; mientras que en las pacientes no gestantes el seguimiento fue durante tres trimestres consecutivos.

Una prueba de equivalencia de medias requirió una muestra de tamaño $n=37$ en el grupo de referencia y en el grupo control, con una potencia de $80 \%$ y una significancia del $5 \%$. La desviación estándar fue de 7-19 y los límites de equivalencia de $-5 a+5$.

Una vez recolectada la información se procedió al vaciamiento de la misma en una base de datos del programa SPSS para su organización, codificación y análisis estadístico propuesto para calcular el análisis logístico para estimar las medidas de tendencia central y dispersión de datos.

\section{Resultados}

Características demográficas

En el grupo gestante se incluyeron 74 ojos en total, de 37 pacientes con diagnóstico de embarazo, todas ingresadas al estudio durante el primer trimestre de la gestación y con seguimiento durante los dos trimestres posteriores para realizar la comparación de las variables: complejo de células ganglionares de la retina y presión intraocular; mientras que en el grupo control se incluyeron 74 ojos en total, de 37 pacientes sanas, todas sin antecedentes patológicos sistémicos u oftalmológicos de importancia. 
En cuanto a la edad de las participantes, en el grupo gestante se obtuvo una media de 25.6 años, con una edad mínima de 18 años y una máxima de 33 años; mientras que en el grupo control la edad promedio fue de 24.2 años, con una edad mínima de 18 años y una máxima de 34 años, sin una diferencia estadísticamente significativa entre ambos grupos $(P=.155)$.

Se realizó la medición de la presión intraocular en ambos grupos en cada visita (una visita por trimestre). En el ojo derecho los promedios para el grupo gestante fueron de $14.4,13.8$ y 13.9 $\mathrm{mm} \mathrm{Hg}$ en el primer, segundo y tercer trimestre de la gestación, respectivamente; mientras que en el grupo control los promedios fueron de 14.0, 14.1 y $14.7 \mathrm{~mm} \mathrm{Hg}$ en el primer, segundo y tercer trimestre de seguimiento, respectivamente (Gráfica 1).

En el ojo izquierdo los promedios para el grupo gestante fueron de $14.4,14.0$ y $13.7 \mathrm{~mm} \mathrm{Hg}$ en el primer, segundo y tercer trimestre de la gestación, respectivamente; mientras que en el grupo control los promedios fueron de 14.1, 14.2 y $14.2 \mathrm{~mm} \mathrm{Hg}$ en el primer, segundo y tercer trimestre de seguimiento, respectivamente (Gráfica 2).
Gráfica 1. Medias de presión intraocular en el ojo derecho

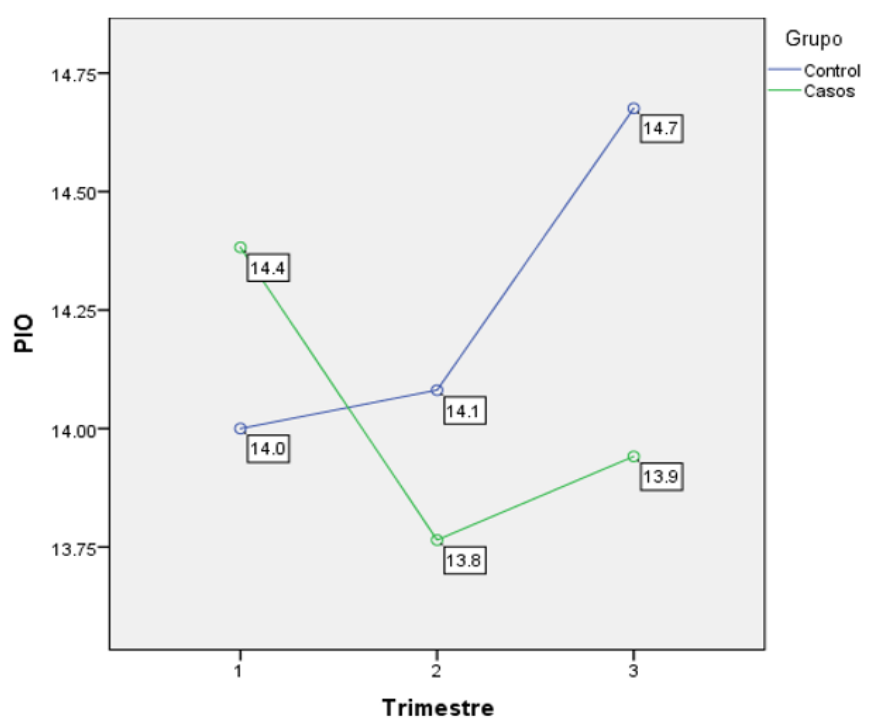

PIO: presión intraocular en $\mathrm{mm} \mathrm{Hg}$.

Gráfica 2. Medias de presión intraocular en el ojo izquierdo

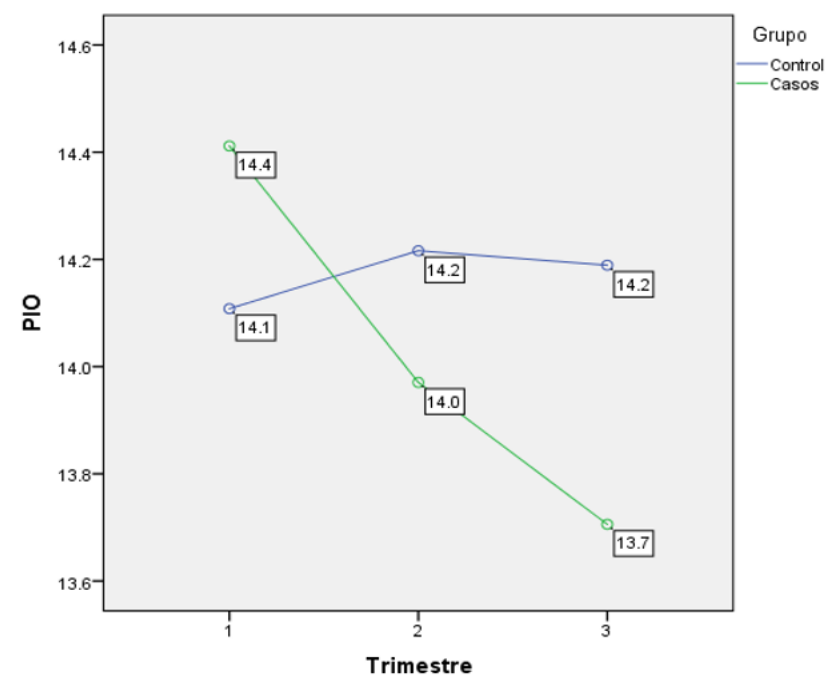

PIO: presión intraocular en $\mathrm{mm} \mathrm{Hg}$.

Respecto a la variable complejo de células ganglionares de la retina en el ojo derecho (OD) los promedios en el grupo gestante fueron de 96.0, 95.7 y 95.1 células durante el primer, segundo y tercer trimestre del embarazo, respectivamente; mientras que en el grupo control los promedios registrados fueron de $96.1,95.8$ y 94.9 células en el primer, segundo y tercer trimestre de seguimiento, respectivamente (Gráfica 3). 
Gráfica 3. Medias del complejo de células ganglionares de la retina en el ojo derecho

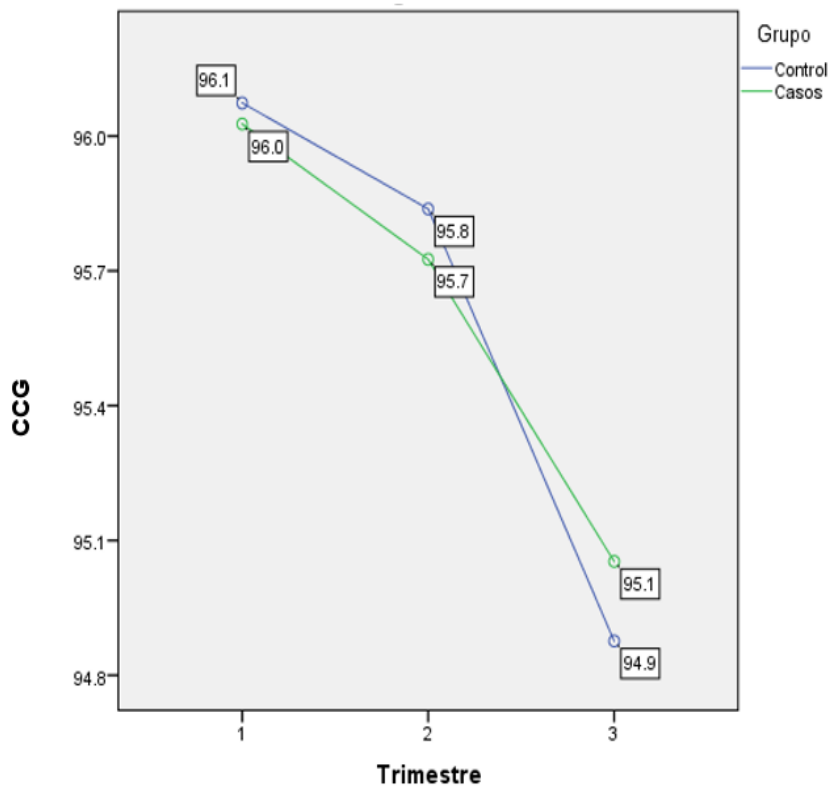

CCG: complejo de células ganglionares de la retina, medido en número de células.

Para el ojo izquierdo (OS) en el grupo gestante se registraron los promedios de 95.7, 95.2 y 94.6 células en el primer, segundo y tercer trimestre de la gestación, respectivamente, mientras que en el grupo control los promedios fueron de 95.8, 95.7 y 94.7 células en el primer, segundo y tercer trimestre de seguimiento, respectivamente (Gráfica 4).

En la tabla 1 se realiza la comparación por parejas de ambos grupos en donde se aprecia que no existe diferencia estadísticamente significativa respecto a la diferencia de medias en ninguna de las variables en ninguno de los ojos.

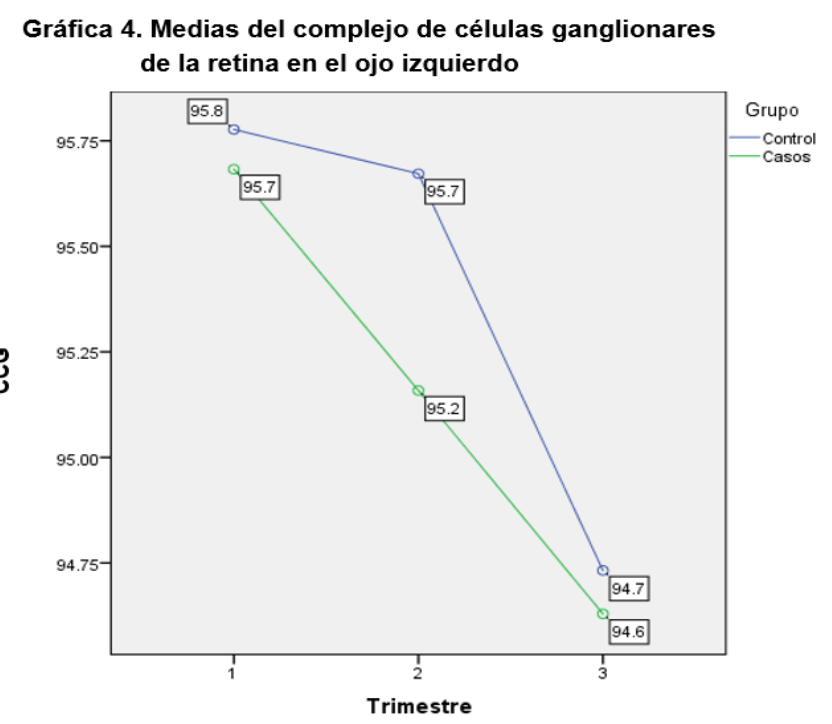

CCG: complejo de células ganglionares de la retina, medido en número de células.

Tabla 1. Comparación por parejas

\begin{tabular}{|c|c|c|c|c|c|}
\hline Medida & $\begin{array}{l}\text { Tri } \\
\text { mes } \\
\text { tre }\end{array}$ & (I) Grupo & (J) Grupo & $\begin{array}{l}\text { Diferenc } \\
\text { ia de } \\
\text { medias } \\
(\mathrm{I}-\mathrm{J})\end{array}$ & Sig. a \\
\hline \multirow[t]{3}{*}{ PIO OD } & 1 & Control & Casos & -.38 & .567 \\
\hline & 2 & Control & Casos & .32 & .545 \\
\hline & 3 & Control & Casos & .73 & .175 \\
\hline \multirow[t]{3}{*}{ PIO OS } & 1 & Control & Casos & -.30 & .671 \\
\hline & 2 & Control & Casos & .25 & .635 \\
\hline & 3 & Control & Casos & .48 & .301 \\
\hline \multirow[t]{3}{*}{ CCG OD } & 1 & Control & Casos & .05 & .973 \\
\hline & 2 & Control & Casos & .11 & .928 \\
\hline & 3 & Control & Casos & -.18 & .895 \\
\hline \multirow[t]{3}{*}{ CCG OS } & & Control & Casos & .09 & .946 \\
\hline & & Control & Casos & .51 & .688 \\
\hline & 3 & Control & Casos & .10 & .937 \\
\hline
\end{tabular}

Se basa en medias marginales estimadas

a. Ajuste para varias comparaciones: menor diferencia significativa (equivalente a sin ajustes). 


\section{Discusión}

El glaucoma es un tipo de neuropatía óptica caracterizada por degeneración progresiva de las células ganglionares de la retina; las cuales constituyen neuronas pertenecientes al sistema nervioso central que tiene sus cuerpos celulares en la porción interna de la retina y cuyos axones forman el nervio óptico. La degeneración de estas células ocasiona una apariencia característica del nervio óptico (palidez, aumento de la excavación, pérdida de la relación copa-disco, etc.) y pérdida irreversible de la visión. La causa de la enfermedad hasta la actualidad aún no es completamente conocida al igual que los factores que la favorecen. ${ }^{7}$

El glaucoma afecta a más de 70 millones de personas en todo el mundo, siendo el responsable de ceguera bilateral en el $10 \%$ de ellos, convirtiéndolo en la principal causa de ceguera irreversible a nivel mundial, de ahí la importancia de un diagnóstico precoz.

Una característica importante de la enfermedad glaucomatosa es que puede permanecer asintomática hasta formas avanzadas, lo que hace con gran frecuencia que las personas con la enfermedad lo desconozcan, siendo aproximadamente sólo del $10-50 \%$ consciente que la padece. $^{8}$

El glaucoma se puede clasificar en dos grandes categorías: glaucoma de ángulo abierto y glaucoma de ángulo cerrado, siendo más frecuente el primero, mientras que el segundo es el responsable de un número desproporcionado de pacientes con pérdida severa de la visión. Ambos tipos de glaucoma se consideran enfermedades primarias. ${ }^{9}$

Existe también el glaucoma secundario el cual puede ser ocasionado por traumas, ciertos medicamentos, inflamación, tumores y algunas patologías como el síndrome de dispersión pigmentaria y el síndrome de pseudoexfoliación. ${ }^{10}$

Una reciente revisión sistemática del JAMA Rational Clinical Examination sobre el glaucoma de ángulo abierto primario describe que para el diagnóstico de glaucoma el riesgo de padecer la enfermedad era más alto cuando a la exploración oftalmoscópica se apreciaba un aumento o asimetría de la relación copa-disco, hemorragia del disco o elevación de la presión intraocular; mientras que el glaucoma primario de ángulo cerrado fue más probable cuando hubo antecedentes familiares, raza negra o edad avanzada. $^{11}$

El único factor que se ha descrito que influye directamente en la evolución del glaucoma es el control de la presión intraocular, por lo cual la base de los tratamientos para la enfermedad se centra en medicamentos hipotensores oculares en sus diferentes variedades (beta bloqueadores, análogos de prostanglandinas, etc. $)^{12}$

Por otra parte, la administración de fármacos durante el embarazo, y especialmente durante 
el primer trimestre de la gestación, siempre es controvertida. Se ha calculado que entre un 60$90 \%$ de las mujeres embarazadas consumen algún fármaco durante la gestación y que hasta un $2-3 \%$ de las malformaciones congénitas son ocasionadas por el consumo de medicamentos.

Hablando específicamente de los tratamientos tópicos oculares la literatura científica disponible es muy limitada; no existen estudios controlados aleatorizados debido a que por razones éticas y legales no se realizan ensayos clínicos en mujeres embarazadas y prácticamente toda la evidencia disponible está basada en casos individuales $o$ en estudios realizados en animales.

Por su parte, todos los fármacos antiglaucomatosos se encuentran dentro de la categoría $C$ farmacológica de la FDA, excepto la brimonidina que es un alfa 2 agonista de categoría B, por lo que sería el más recomendable en este grupo de pacientes.

Como norma general, habría que evitar todos los fármacos durante el primer trimestre de la gestación, ya que es el periodo de organogénesis siendo mayor el riesgo de teratogenicidad. En caso de necesidad se recomienda el uso de fármacos con nivel de seguridad A, B o C, siempre valorando los riesgos y beneficios del tratamiento. ${ }^{13}$

La administración de fármacos oculares en las mujeres embarazadas conlleva en general un riesgo fetal bajo, debido a que sólo la fórmula no ionizada de las moléculas solubles, que son las más utilizadas en oftalmología, penetra en el epitelio corneal, por lo que únicamente del 1-5\% logra alcanzar el humor acuoso.

El uso de beta bloqueadores tópicos como el timolol suele ser frecuente en pacientes gestantes debido a la experiencia clínica con este grupo de fármacos; sin embargo, existen casos descritos de bradicardia y arritmia fetal tras la administración de timolol en gotas oftálmicas y se sabe que alcanza concentraciones en la leche materna. ${ }^{14}$

Respecto al glaucoma durante la gestación, se ha descrito que existe una disminución en la progresión de la enfermedad, permitiendo la suspensión de los medicamentos hipotensores oculares durante el embarazo, evitando así la exposición del embrión a los mismos y sus potenciales efectos adversos. ${ }^{15}$

En este estudio se pretendió comparar los valores del complejo de células ganglionares de la retina y la presión intraocular en cada uno de los trimestres de la gestación en pacientes gestantes sanas (debido a la poca prevalencia del glaucoma en el embarazo) con pacientes no gestantes también sanas, quienes se valoraron durante tres trimestres consecutivos. 


\section{Conclusiones}

Nuestro estudio arrojó resultados que difieren con lo descrito por otros autores respecto a la presión intraocular durante la gestación ya que no se encontraron diferencia clínicas ni estadísticamente significativas en las mediciones realizadas en ambos grupos.

El complejo de células ganglionares de la retina permaneció dentro de parámetros normales durante cada uno de los trimestres de la gestación y $\sin$ cambios estadísticamente significativos respecto al grupo control.

Este estudio presenta limitaciones y una de ellas es que las pacientes estudiadas no contaban con diagnóstico de patología glaucomatosa (en ninguno de los dos grupos) lo cual hace que los resultados no sean extrapolables a un grupo con la patología, pero permiten conocer la evolución del complejo de células ganglionares de la retina y la descripción de la presión intraocular durante la gestación lo cual puede ser utilizado como una base normativa.

La ventaja de nuestro estudio es que existen pocos análisis realizados en mujeres embarazadas y menos aún que cuenten con el seguimiento completo de la gestación estudiando el comportamiento del complejo de células ganglionares de la retina y la presión intraocular para de esta manera lograr realizar una comparación de dichos parámetros con mujeres sanas no gestantes durante un mismo periodo de tiempo.

Los resultados obtenidos pueden abrir una puerta a más estudios que valoren los parámetros antes mencionados en comparación con los registrados en pacientes con glaucoma y de esta manera poder llevar un mejor control y toma de decisiones referentes a la enfermedad durante este específico y frágil periodo que es la gestación.

\section{Referencias}

1. Hamilton WD, Henderson PA, Moran NA. The pathophysiology and treatment of glaucoma: a review. Nat Sel Soc Behav (Eds $\mathrm{R}$ D Alexander D W Tinkle), Chiron Press New York. 1981;311(18):363-81

2. American Academy of Ophthalmology. Glaucoma: Introduction to Glaucoma. Am Acad Ophthalmol. 2011;24-7.

3. Addepalli UK, Jonnadula GB, Garudadri CS, Rao HL, Rani PK, Chakrabarti S, et al. LV Prasad eye institute glaucoma epidemiology and molecular genetic study (LVPEIGLEAMS). Report 1: Study design and research methodology. Ophthalmic Epidemiol. 2013;20(3):188-95.

4. Cook C, Foster P. Epidemiology of glaucoma: What's new? Can J Ophthalmol [Internet]. 2012;47(3):223-6. Available from: http://dx.doi.org/10.1016/j.jcjo.2012.02.00.

5. Cohen LP, Pasquale LR. Clinical characteristics and current treatment of glaucoma. Cold Spring Harb Perspect Med. 2014;4(6). 
6. Shin $\mathrm{YU}$, Hong EH, Kang $\mathrm{MH}$, Cho H, Seong $\mathrm{M}$. The Association between Female Reproductive Factors and Open-Angle Glaucoma in Korean Women: The Korean National Health and Nutrition Examination Survey V. 2018;2018.

7. Ahmad SS. Glaucoma suspect: A Pratical Approach. Taiwan J Ophthalmol. 2017;8:535.

8. Serna-Ojeda JC, Flores-Reyes EM, Hartleben-Matkin C. Glaucoma: Corrientes en estudio sobre su etiología. Gac Med Mex. 2014;150:317-23.

9. Diez RC, Román JJ, Barbosab MJl. Concepto de sospecha de glaucoma de ángulo abierto: Definición, diagnóstico y tratamiento. Rev Mex Oftalmol. 2014;88(4):15360 .

10. Likhvantseva VG, Sokolov VA, Levanova ON K. Predicting the probability of development and progression of primary open angle glaucoma by regression modeling. Vestn Oftalmol. 2018;134(3):35-41.
11. Hollands, H., Johnson, D., Hollands, S., Simel, D. L., Jinapriya, D., \& Sharma, S. (2013). Do findings on routine examination identify patients at risk for primary open-angle glaucoma?: The rational clinical examination systematic review. JAMA, 309(19), 2035-2042.

12.Guglielmi P, Carradori S, Campestre C, Poce G. Novel therapies for glaucoma: a patent review (2013-2019). Expert Opin Ther Pat [Internet]. 2019;29(10):769-80.

13. Savino PJ, Johnson SM, Martinez M, Freedman S. Management of Glaucoma in Pregnancy and Lactation. 2001;45(5).

14.Pasquale LR, Grosskreutz CL. The Course of Glaucoma During Pregnancy. Arch Ophthalmol. 2016;124(1):1089-94.

15. Drake, S. C., \& Vajaranant, T. S. (2016). Evidence-based approaches to glaucoma management during pregnancy and lactation. Current ophthalmology reports, 4(4), 198205. 